\title{
To Make Modeling a Natural Tool in Business Development We Need to Stop Talking about Modeling
}

\author{
Christer Nellborn \\ Nellborn Management Consulting AB \\ Spiselvägen 14 \\ 14159 Huddinge, Sweden \\ christer@nellborn.se \\ www. nellborn. se
}

\begin{abstract}
To make enterprise modeling a natural tool for business development we need to integrate with other disciplines such as sociology, psychology and economy. We also need to focus more on the business problems to solve and the benefits and effects the business gets from using the models and less on the models themselves.
\end{abstract}

Keywords: Business modeling, enterprise modeling.

\section{Background}

Business modeling is nothing new. It has been around for a very long time. Some of the earliest documented examples can be found in the drawings our cavemen ancestors made of animals being hunted. Dressed in modern terminology these cave drawings are business models showing how to optimize the utilization of available enterprise resources in a gathering process in order to maximize the achievement of important short- and long term business goals - i.e. kill the beast to get food to eat and material for making clothes to keep us warm at winter.

Since the early stages of the industrial era, a lot of research and development has been put into developing models and techniques for analyzing and handling the complexity when enterprises grow from small and local to large and international. These models come from a wide variety of disciplines such as economy, psychology and sociology.

In the last 30 or so years, automated information systems have emerged as a crucial tool for organizations to manage complexity. One of the key questions for information systems development is getting good, sound, complete requirements. This has been a challenge for many years and proven to be a very elusive problem. Many models, techniques, tools and approaches have been developed to handle the problem.

\section{Business Development and Models}

Business development can be seen as consisting of three areas:

1. The development of the relations and interactions between the organization and its surroundings, i.e. customers, suppliers, competitors, society in general 
2. The development of resources and how the organization utilize them to support the external interactions

3. The development of computerized business resources

A significant difference between the first two and the third area is the implementation of changes. For the first two, the implementation is mainly in the way people in the organization work, interact and behave, whereas the implementation in the third area is mainly in the functions of a computer system.

The purposes of models for the three areas are therefor slightly different. When implementing changes in a computer system you need models for designing the system, preferably with as much detail as possible to remove guesswork and to get precise requirements.

When implementing changes in the behavior of people you need models that support communication, learning and understanding. Models for this purpose need to focus more on context, reason and motivation and less on detailed design of the organization. People will work out the details for themselves in discussions, interactions and in their practical day-by-day work.

\section{The Proof of the Pudding Is in the Eating}

To succeed in making enterprise modeling a natural tool in business development, we need to do three things:

1. Stop talking about the models and the modeling as such. Focus on how the models can support the change process

2. Integrate more with the traditional disciplines for enterprise modeling (i.e. economy, sociology, psychology et.c.). Cooperate in the development of new enterprise models.

3. Make models easier to use in communication with people without technical background. If people don't understand the models they will not use them no matter how good they are. 